\title{
Gastric Mixed Adenoneuroendocrine Carcinoma with Squamous Differentiation: A Case Report
}

\author{
Han-Ik Bae · Chaeyoon Lee ${ }^{1}$ Y Young-Min Jo · Ohkyung Kwon ${ }^{1}$. Wansik Yu ${ }^{1}$ Mee-Seon Kim² · An Na Seo \\ Department of Pathology, Kyungpook National University Hospital, Kyungpook National University Medical Center, Kyungpook National University School of Medicine, Daegu; \\ 'Department of Surgery, Gastric Cancer Center, Kyungpook National University Medical Center, Kyungpook National University School of Medicine, Daegu; \\ ${ }^{2}$ Department of Pathology, Samsung Changwon Hospital, Sungkyunkwan University School of Medicine, Changwon, Korea
}

The occurrence of gastric mixed adenoneuroendocrine carcinoma (MANEC) is relatively rare, although a few cells of gastric adenocarcinoma frequently show neuroendocrine differentiation. ${ }^{1}$

We herein present one of only a few reported cases of gastric MANEC with trilineage histologic differentiation, composed of adenocarcinoma, large cell neuroendocrine carcinoma (NEC), and squamous cell carcinoma (SqCC).

\section{CASE REPORT}

A 60-year-old woman presented with generalized weakness, anorexia, weight loss, and fasting epigastric pain over a 5-month period. The patient had suffered from allergic urticaria for several years, but this had been treated and subsequently controlled with standard medication. She denied any previous alcohol or herbal medication intake and had no significant family history. Esophagogastroduodenoscopy revealed an $8.5 \times 7.0-\mathrm{cm}$-sized ulcerofungating mass in the anterior wall of the lower body. Biopsy of the mass was diagnosed as moderately differentiated adenocarcinoma. Preliminary laboratory test results were within normal limits, whereas a tumor marker level test revealed elevat-

\section{Corresponding Author}

An Na Seo, MD, PhD

Department of Pathology, Kyungpook National University Hospital, Kyungpook National University Medical Center, Kyungpook National University School of Medicine, 807 Hoguk-ro, Buk-gu, Daegu 41404, Korea

Tel: +82-53-200-3403, Fax: +82-53-200-3399, E-mail: san_0729@naver.com

Mee-Seon Kim, MD

Department of Pathology, Samsung Changwon Hospital, Sungkyunkwan University School of Medicine, 158 Paryong-ro, Masanhoewon-gu, Changwon 51353, Korea Tel: +82-55-290-1332, Fax: +82-55-290-6148, E-mail: kimm23@naver.com

Received: September 22, 2015 Revised: October 9, 2015

Accepted: October 15, 2015 ed carbohydrate antigen 19-9 (98.8 U/mL). Computed tomography demonstrated a large hypodense ulcerofungating mass in the gastric anterior wall along with multiple perigastric lymphadenopathies (Fig. 1A). The patient underwent D2 subtotal gastrectomy and early postoperative intraperitoneal chemotherapy. Gross examination of the resected stomach showed a smooth serosal surface and a large ulcerofungating mass measuring 8.5 $\times 7.0 \mathrm{~cm}$, consistent with Borrmann type 2, in the lower anterior wall (Fig. 1B). On sectioning, the tumor had a heterogeneous appearance with a mixture of white and yellow lesions with infiltrative boundaries, invading to the serosal layer (Fig. 1C). Microscopically, the tumor comprised 55\% large cell NEC, 35\% moderately differentiated adenocarcinoma, and $10 \%$ well differentiated SqCC. The adenocarcinoma component was in the upper portion of the tumor, whereas the NEC component presented in the deep portion, with a small proportion in the upper portion, up to the mucosal layer (Fig. 1D). In particular, the collision between the adenocarcinoma and NEC components was found in the ulcerated tumor area (Fig. 2A). Additionally, transition from NEC to SqCC was found in some areas (Fig. 2C). As expected, immunohistochemical staining for synaptophysin and chromogranin A were positive in the NEC component but negative in the adenocarcinoma and SqCC components (Fig. 2B, D). The SqCC component but not the NEC or adenocarcinoma component revealed strong nuclear expression for p63 (Fig. 2E). The tumor mitotic count was 95 mitoses per 10 high-power fields, and the Ki-67 labeling index was 96\%. The tumor penetrated the serosa, and 14 of the 48 regional lymph nodes showed metastasis, which, interestingly, was composed of only the adenocarcinoma component (Fig. 2F), but distant metastasis was not detected. Interestingly, for c-erbB2, the NEC com- 

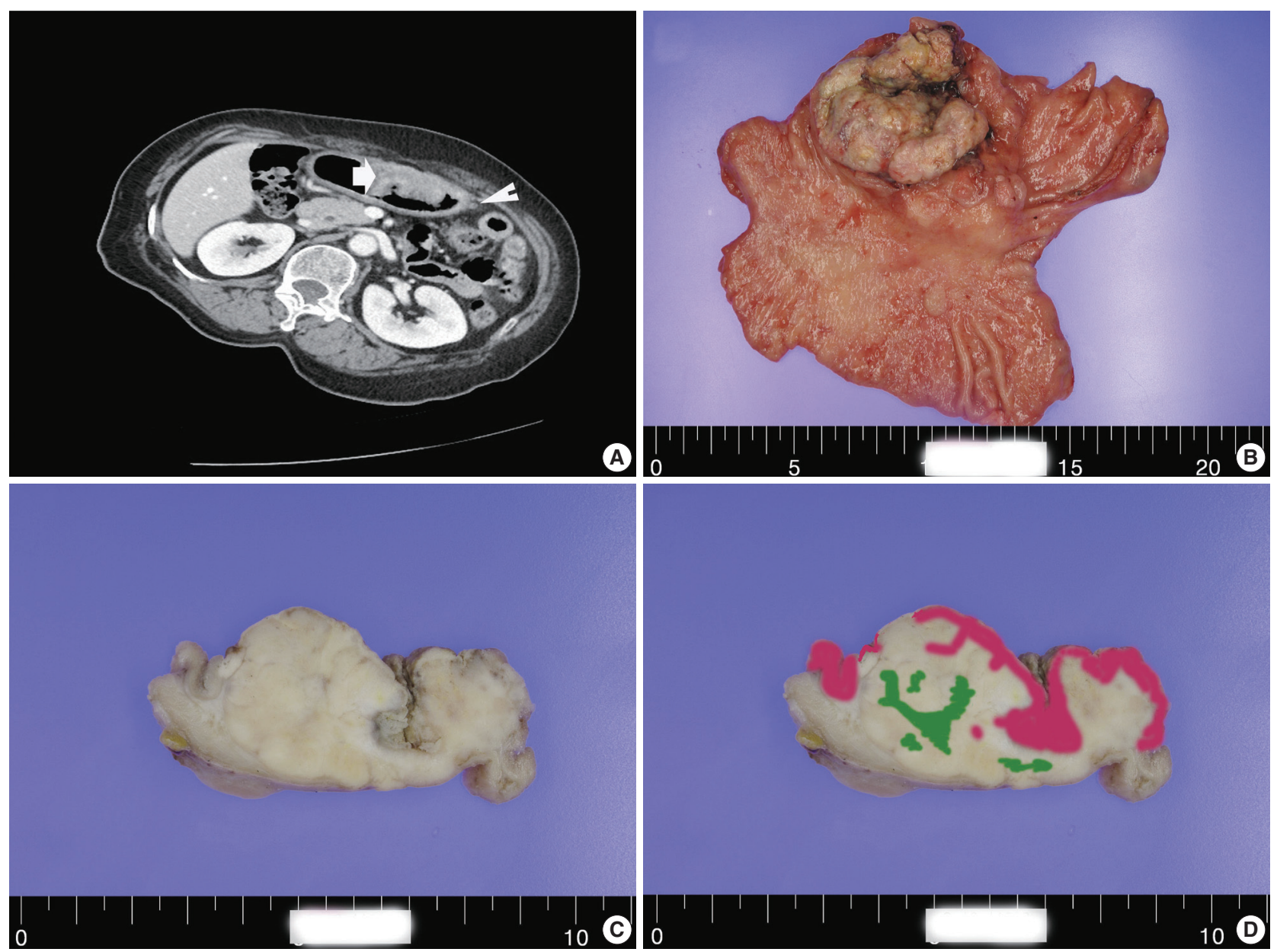

Fig. 1. (A) A computed tomography scan demonstrating a large ulcerofungating hypodense mass in the anterior gastric wall (arrow) and enlargement of multiple perigastric lymph nodes (arrowhead). Macroscopic findings (B) and cross-section of gastric cancer (C). (D) Mapping of the gastric mixed adenoneuroendocrine carcinoma with trilineage histologic differentiation composed of adenocarcinoma (pink color), large cell neuroendocrine carcinoma, and squamous cell carcinoma (green color).

ponent revealed negativity, whereas the adenocarcinoma component showed complete membranous staining of strong intensity in primary gastric cancer and in its corresponding metastatic regional lymph nodes (Fig. 3). During seven cycles of adjuvant chemotherapy, the patient experienced sudden abdominal pain due to mechanical obstruction by an adhesion at the operation site. Since then, she has stopped receiving chemotherapy and is currently receiving supportive care. The patient has been monitored for 18 months post-surgery and remains alive without evidence of disease recurrence.

The study was performed in accordance with the guidelines of the Institutional Review Board of Kyungpook National University Medical Center and was exempt from written informed consent. The information of this case was retrospectively obtained from medical records in the database of Kyungpook National University Medical Center.

\section{DISCUSSION}

Gastric MANECs are mixed exocrine and endocrine carcinomas, with one component exceeding at least $30 \%$ of the tumor lesion. ${ }^{1,2}$ The exocrine component is characterized by adenocarcinoma with various differentiated grades, and the neuroendocrine component is usually represented by NEC. ${ }^{1}$ To our knowledge, there have been only four reported cases of gastric MANEC containing a SqCC component. ${ }^{3-5}$ Pericleous et al. ${ }^{5}$ reported a case of MANEC with a SqCC component where only the NEC component metastasized to the liver. In addition, Shibuya $e t$ al. ${ }^{4}$ reported a small cell undifferentiated carcinoma with adenocarcinoma and SqCC components, and hepatic nodules showed an atypical carcinoid component. In contrast, in the current case, only the adenocarcinoma component metastasized to the regional lymph nodes, with no distant metastasis detected. 
The pathogenesis of gastric NEC is unclear. Based on the presence of NEC with other glandular or squamous cell components, the proposed origin of gastric MANEC is pluripotent stem cells that have the potential to differentiate into other cell types producing mucin or keratin. ${ }^{6,7}$ Owing to the heterogeneous composition of MANEC, treatment approaches should focus on the
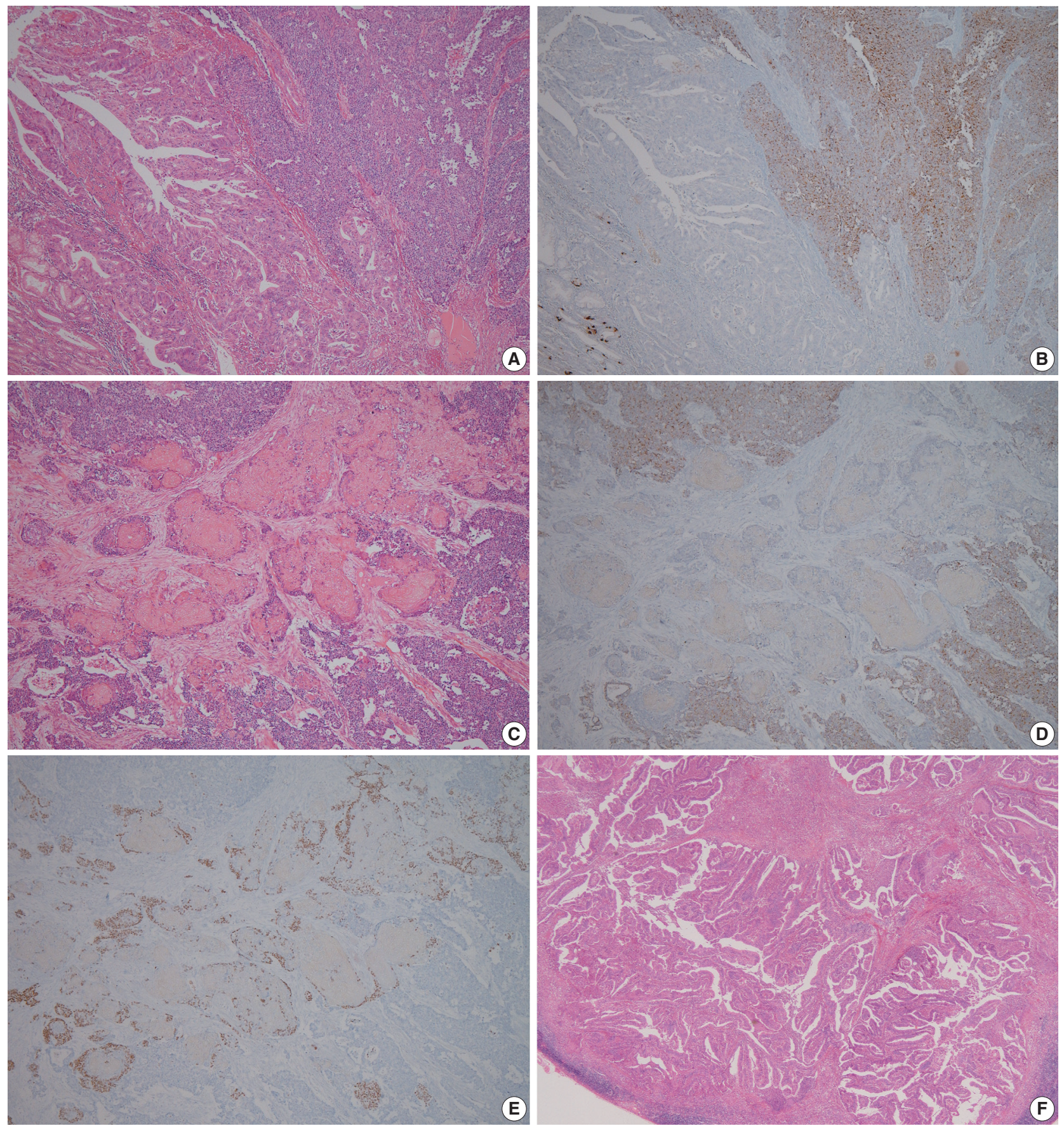

Fig. 2. Representative microscopic findings of mixed adenoneuroendocrine carcinoma with trilineage histologic differentiation. (A) Moderately differentiated adenocarcinoma admixed with large cell neuroendocrine carcinoma (NEC). (B) The NEC component demonstrates positive staining for chromogranin A, whereas the adenocarcinoma component shows negativity. (C) Squamous cell carcinoma (SqCC) component admixed with the NEC component, and (D) the NEC component presented positivity for chromogranin A, but not the SqCC component. (E) The cells with squamous cell differentiation are identified by expression of p63. (F) Metastasis of only the adenocarcinoma component to the gastric regional lymph nodes. 

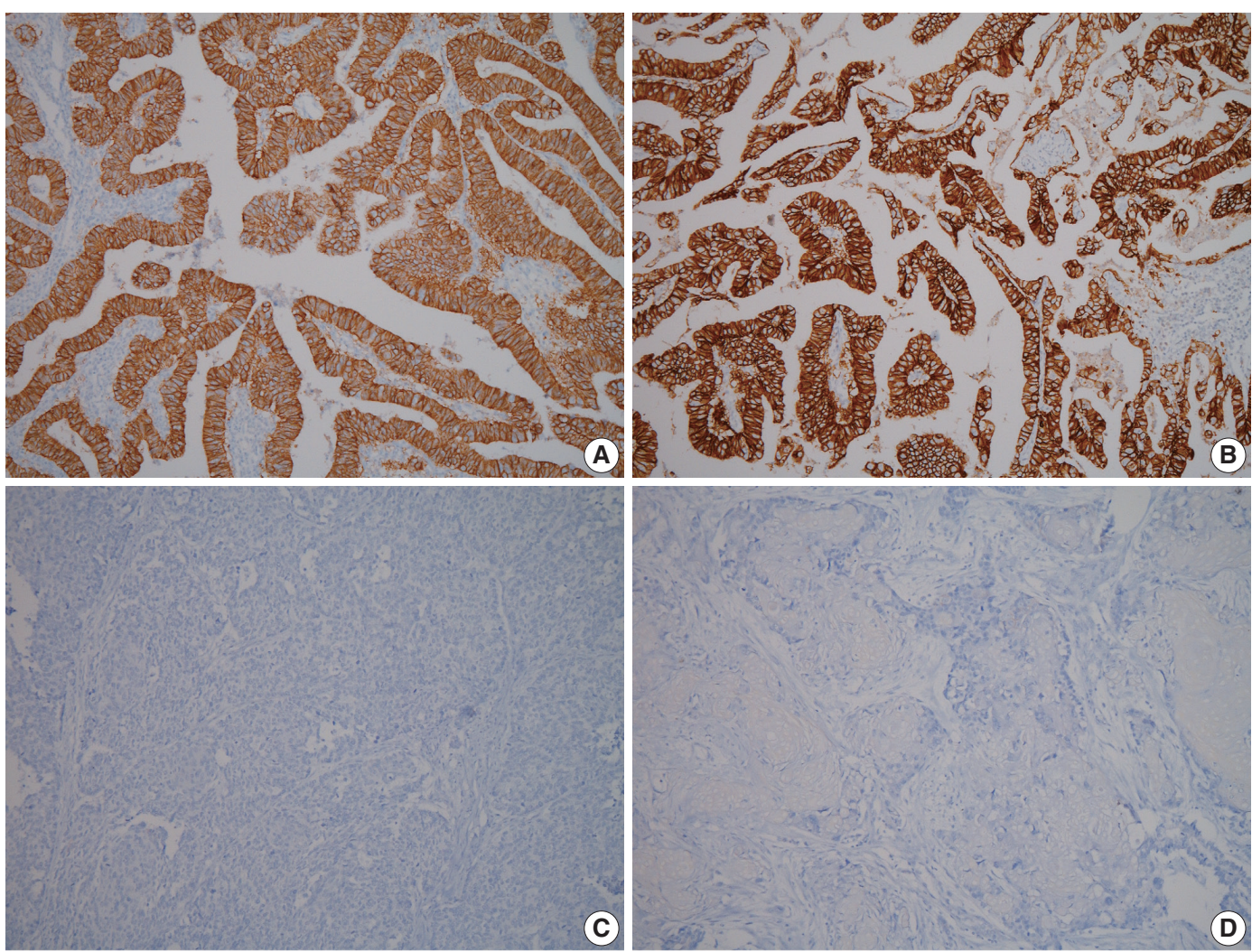

Fig. 3. Photomicrographs of immunohistochemistry $(\mathrm{HC})$ for c-erbB2 in mixed adenoneuroendocrine carcinoma with trilineage histologic differentiation. $\mathrm{IHC}$ for $\mathrm{c}$-erbB2 shows strong positivity in the adenocarcinoma component (A), and in the metastatic regional lymph nodes (B), whereas $\mathrm{HC}$ for c-erbB2 is negative in neuroendocrine carcinoma $(\mathrm{C})$ and squamous cell carcinoma component $(\mathrm{D})$.

most aggressive tumor components. However, the prognosis and behavior of gastric MANEC with trilineage histologic differentiation are unknown because of its rarity. Therefore, collaborative multicenter studies including such patients are required to determine optimal treatment regimens, prevent relapse, and improve prognosis.

Herein, we report a gastric MANEC with trilineage histologic differentiation including large cell NEC, adenocarcinoma, and SqCC, of which only the adenocarcinoma component metastasized to the regional lymph nodes, without distant metastasis.

\section{Conflicts of Interest}

No potential conflict of interest relevant to this article was reported.

\section{REFERENCES}

1. Bosman FT, Carneiro F, Hruban RH, Theise ND. WHO classification of tumours of the digestive system. 4th ed. Lyon: IARC Press, 2010.
2. Volante M, Rindi G, Papotti M. The grey zone between pure (neuro)endocrine and non-(neuro)endocrine tumours: a comment on concepts and classification of mixed exocrine-endocrine neoplasms. Virchows Arch 2006; 449: 499-506.

3. Haratake J, Horie A, Inoshita S. Gastric small cell carcinoma with squamous and neuroendocrine differentiation. Pathology 1992; 24: 116-20.

4. Shibuya H, Azumi N, Abe F. Gastric small-cell undifferentiated carcinoma with adeno and squamous cell carcinoma components. Acta Pathol Jpn 1985; 35: 473-80.

5. Pericleous M, Toumpanakis C, Lumgair $\mathrm{H}$, et al. Gastric mixed adenoneuroendocrine carcinoma with a trilineage cell differentiation: case report and review of the literature. Case Rep Oncol 2012; 5: 313-9.

6. Bartley AN, Rashid A, Fournier KF, Abraham SC. Neuroendocrine and mucinous differentiation in signet ring cell carcinoma of the stomach: evidence for a common cell of origin in composite tumors. Hum Pathol 2011; 42: 1420-9.

7. Kim KM, Kim MJ, Cho BK, Choi SW, Rhyu MG. Genetic evidence for the multi-step progression of mixed glandular-neuroendocrine gastric carcinomas. Virchows Arch 2002; 440: 85-93. 